\title{
Percutaneous Medial Collateral Ligament Release Improves Medial Compartment Access During Knee Arthroscopy
}

\author{
Thomas E. Moran, M.D., Alex J. Demers, B.S., Kaitlyn M. Shank, Ed.A.T.C., \\ John T. Awowale, M.D., and Mark D. Miller, M.D.
}

\begin{abstract}
Purpose: To quantify intraoperative joint space widening afforded by the outside-in, percutaneous release of the medial collateral ligament (MCL) and to evaluate its impact on medial compartment width and functional outcomes at 6-week follow-up for patients undergoing a partial medial meniscectomy without postoperative bracing. Methods: Patients with posteromedial meniscus tears and no evidence of ipsilateral knee pathology, undergoing partial medial meniscectomy, were enrolled. Intraoperatively, medial compartment width was quantified with fluoroscopy before and after the percutaneous MCL release with an 18-gauge spinal needle proximal to the joint line. At 6-week follow-up, valgus stress radiographs re-evaluated medial compartment width. International Knee Documentation Committee (IKDC) and PatientReported Outcomes Measurement Information System (PROMIS) scores were completed preoperatively and at 6-week follow-up to evaluate functional outcomes. A paired sample $t$ test performed at a $95 \%$ confidence interval (CI) was used to compare these variables. Results: Forty-two patients, mean ( \pm standard deviation) age $55.3 \pm 10.7$ years, were available for analysis of intraoperative medial compartment widening. Medial compartment width increased from $5.95 \pm$ 1.32 to $11.09 \pm 1.74 \mathrm{~mm}$ intraoperatively after MCL release. At 6-week follow-up, radiographic assessment demonstrated a mean medial compartment width of $5.85 \pm .99 \mathrm{~mm}$, which represented an insignificant change compared with the preoperative value (CI -0.68 to $.33, P=.474)$. PROMIS and IKDC scores significantly improved from baseline, with increases of $6.9 \pm 12.4$ (CI 2.0 to $11.8, P=.008$ ) and $11.7 \pm 17.8$ (CI 4.7 to $18.8, P=.002$ ), respectively. Conclusions: Percutaneous MCL release during knee arthroscopy improves visualization and facilitates instrumentation by providing an almost $2 \times$ wider working space within the medial tibiofemoral joint. In this study, the performance of percutaneous MCL release did not result in any complications. Radiographic and clinical resolution of iatrogenic laxity was demonstrated by 6-weeks postoperatively, without the use of postoperative bracing. Level of Evidence: IV, therapeutic case series.
\end{abstract}

$\mathbf{K}$ nee arthroscopy is the most common surgery performed to treat injuries to the meniscus. ${ }^{1}$

From the Department of Orthopaedic Surgery, University of Virginia, Charlottesville, Virginia, U.S.A.

The authors report the following potential conflicts of interest or sources of funding: M.D.M. reports other, Arthrex; board or committee member, American Orthopaedic Society for Sports Medicine, Miller Orthopaedic Review Enterprises; publishing royalties, financial or material support, Saunders/ Mosby, Elsevier, Wolter Kluwer Health, Lippincott Williams $\theta$ Wilkins. Full ICMJE author disclosure forms are available for this article online, as supplementary material.

Received March 27, 2020; accepted August 25, 2020.

Address correspondence to Dr. Mark D. Miller, M.D., 515 Ray C. Hunt Dr, Suite 1100, Charlottesville,VA 22903,U.S.A.E-mail:mdm3p@virginia.edu

(C) 2020 THE AUTHORS. Published by Elsevier Inc. on behalf of the Arthroscopy Association of North America. This is an open access article under the CC BY-NC-ND license (http://creativecommons.org/licenses/by-nc-nd/4.0/). 2666-061X/20407

https://doi.org/10.1016/j.asmr.2020.08.014
Adequate arthroscopic examination and surgical intervention to address pathology of the knee require complete visualization of the intra-articular anatomy. ${ }^{2}$ Certain areas of the knee can be challenging to access for full visualization or perform interventions without causing iatrogenic cartilage damage, which is the most common complication from knee arthroscopy. ${ }^{3}$

Additionally, a lack of sufficient working space for instrumentation can increase operative time and prevent optimal clinical results from being obtained through failure to recognize the presence or extent of meniscal pathology. Despite being underreported, these metrics are potentially more common than previously believed. $^{3}$ The posterior medial compartment is an area that has been identified as being particularly difficult to completely visualize, especially in patients with tight medial compartments of the knee. ${ }^{4,5}$ In such cases, the application of valgus stress while the knee is held in $30^{\circ}$ 
flexion can be attempted to increase the working space of this region. This technique, however, is sometimes insufficient in allowing adequate visualization or instrumentation to occur.

In such cases, the senior author's preferred method of the senior author (M.D.M.) to improve the working space of the posterior medial compartment of the knee is to perform an outside-in, percutaneous release of the superficial medial collateral ligament (MCL) to increase access to this region of the joint. Several studies have described this technique for this purpose, and a recently published systematic review concluded that this technique was an effective means of increasing the medial tibiofemoral joint space without causing residual valgus laxity, pain, loss of function, or damage to the saphenous nerve or greater saphenous vein. ${ }^{6-12}$ Despite the conclusions drawn from previous studies and the senior author's experience that this is an effective method of increasing the medial tibiofemoral joint space, there has been only 1 study of 18 patients quantifying medial compartment opening intraoperatively immediately after the release is performed. ${ }^{13}$ It also remains unclear how long any iatrogenic laxity takes to resolve or whether bracing is required postoperatively.

The purpose of this study was to quantify intraoperative joint space widening afforded by outside-in, percutaneous release of the superficial medial collateral ligament (MCL) and to evaluate its impact on medial compartment width and functional outcomes at 6-week follow-up for patients undergoing partial medial meniscectomy without postoperative bracing. We hypothesized that the outside-in, percutaneous release of the medial collateral ligament would produce a significant increase in the medial joint space intraoperatively and demonstrate radiographic and clinical resolution of any iatrogenic knee laxity by 6 weeks postoperatively.

\section{Methods}

\section{Patients}

This prospective study was approved by the academic center's Institutional Review Board, and all identified patients $(n=59)$ provided informed consent. All arthroscopic surgeries were performed between April 2019 and March 2020 by the senior author (M.D.M.). Patients met the criteria for inclusion in this study if they were identified as having an isolated posteromedial meniscus tear with a suspected need for improved visualization and access of instrumentation in the posteromedial compartment, for which they electively wished to pursue partial medial meniscectomy. Inclusion criteria also required patients to have no preexisting varus or valgus laxity in the operative knee, as identified through performance of a varus-valgus stress test with the knee in $0^{\circ}$ and $30^{\circ}$ flexion. Patients were

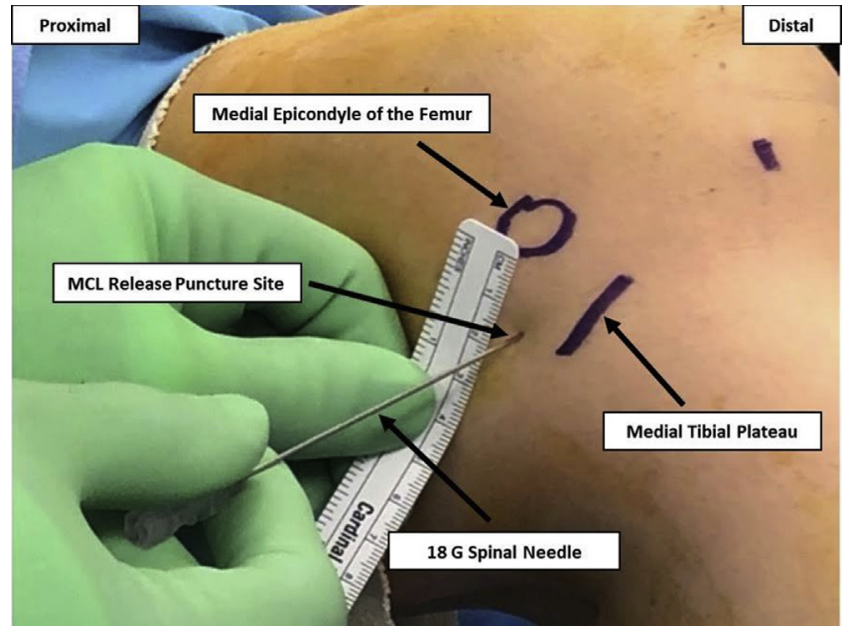

Fig 1. Landmarks are used to identify the point in which percutaneous release of the medial collateral ligament (MCL) is to be performed. The point may be identified $1.5 \mathrm{~cm}$ posterior and slightly distal to the medial epicondyle.

excluded if they had concomitant or associated ipsilateral ligamentous injuries, previous ligamentous reconstruction surgery in the ipsilateral knee, malalignment $>5 \cdot$, or Kellgren-Lawrence grade 3 to 4 arthrosis in the operative knee.

Patients who consented to participate in this study were given validated, baseline questionnaires examining the functional status of the knee. ${ }^{14}$ PatientReported Outcomes Measurement Information System (PROMIS) scores and International Knee Documentation Committee (IKDC) scores were collected from subjects in person during the preoperative clinical visit.

\section{Surgical Technique}

All procedures were performed with the patient supine on the operating room table, with a lateral arthroscopic stress post in place and a nonsterile, pneumatic tourniquet positioned on the patient's thigh. A standard anterolateral viewing portal and anteromedial instrumentation portal were used, and a $4.0-\mathrm{mm}, 30^{\circ}$ arthroscope was used to inspect the intraarticular anatomy in the standard manner for a diagnostic knee arthroscopy. ${ }^{2}$ Intraoperatively, if it was determined that medial access was restricted, an intraoperative valgus stress fluoroscopic image was taken with the aperture of a hemostat handle as a standard sizer. For acquisition of the stress image, a valgus force was applied to the knee by the surgeon through displacement of the distal lower extremity laterally at the level of the medial malleolus with the surgeon's body weight, while a counteracting normal force was applied at the joint line with the surgeon's hand in a solid sterile metal socket. The lower extremity was laterally displaced to a point at which the stress placed on the MCL was at a subjective maximum that was deemed not to cause rupture of the ligament. The knee 


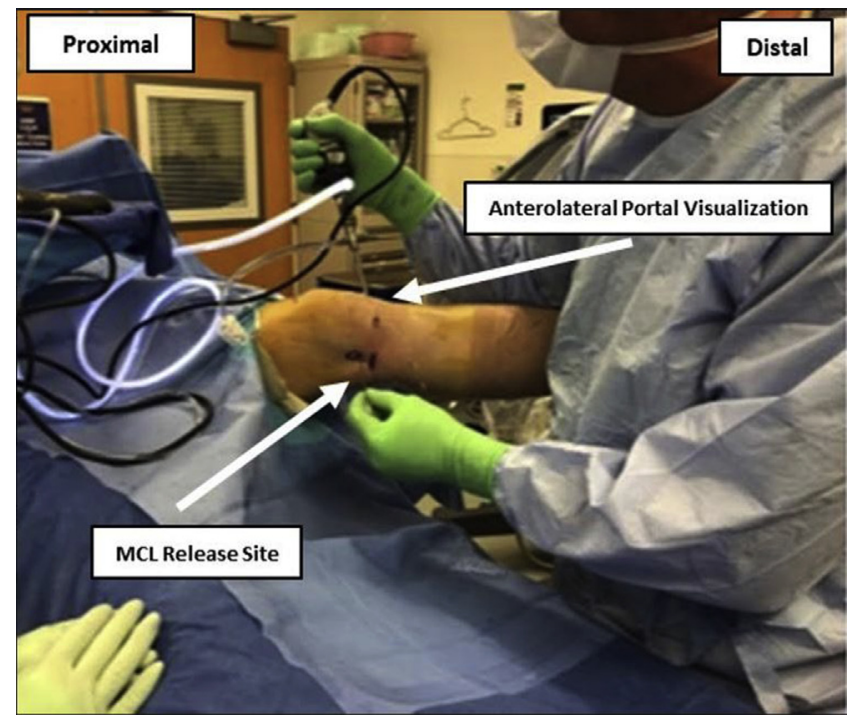

Fig 2. To perform percutaneous release of the medial collateral ligament (MCL), a constant valgus force is applied to the knee while an 18-gauge spinal needle is used to penetrate the skin and fenestrate the superficial MCL in an anterior to posterior direction.

was subsequently drained of fluid, and a C-arm was aligned to obtain an anterior-posterior fluoroscopic image of the knee. The reference hemostat was held by the surgeon at the working end of the instrument longitudinally with the long axis in parallel with the joint space and level with the floor.

To perform the MCL release, a point was identified $1.5 \mathrm{~cm}$ posterior and slightly distal to the medial epicondyle (Fig 1). With a constant valgus force applied to the knee, an 18-gauge spinal needle was used to penetrate the skin and fenestrate the superficial MCL with rapid anterior-to-posterior translation until an audible pop was heard to suggest the increase of the medial compartment width (Fig 2). Simultaneous intraarticular visualization was provided to confirm MCL release through the anterolateral portal (Fig 3). Next, another valgus stress fluoroscopic image with the hemostat as a standard sizer was obtained according to the above protocol. The fluid was again drained from the knee before acquisition of the postrelease intraoperative valgus-stress radiograph.

To ensure consistent measurements regardless of variation in fluoroscopic magnification, a hemostat handle with known aperture minor axis of $2 \mathrm{~cm}$ was used as a reference tool (Fig 4). Postoperatively, the medial compartment joint space was quantified by the senior author (M.D.M.) through the manual ruler measurement of the line created by the medialmost point of the tibial plateau and femoral condyle defining the joint space on the printed fluoroscopic image. The minor axis of the hemostat aperture in the printed fluoroscopic image was also measured using the ruler. The measured joint space of the fluoroscopic image was then multiplied by the magnification conversion factor of $2 \mathrm{~cm}$ divided by the measured value of the hemostat aperture minor axis to produce the width of the medial compartment adjusted for magnification in the fluoroscopic image. This was performed on the fluoroscopic images obtained before and after the performance of the release to determine the additional medial joint space opening provided by the release (Fig 5).

After the percutaneous MCL release, the indicated partial medial meniscectomy procedure was performed. After surgery, patients underwent the institution's standard postoperative protocol without a brace. Patients were allowed to bear weight as tolerated, and a series of progressive exercises were provided to the patients to improve range of motion and strengthen the quadriceps muscles.

Patients returned for follow-up evaluation in clinic 2 and 6 weeks postoperatively. At 6 weeks, patients were again assessed using PROMIS and IKDC questionnaires, and follow-up valgus stress radiographs were acquired to quantify the medial compartment joint space. Patients were also evaluated for evidence of greater

Fig 3. Percutaneous medial collateral ligament (MCL) release is performed under arthroscopic visualization. Intra-articular visualization through the anterolateral portal while the percutaneous MCL release is performed allows confirmation of adequate release.

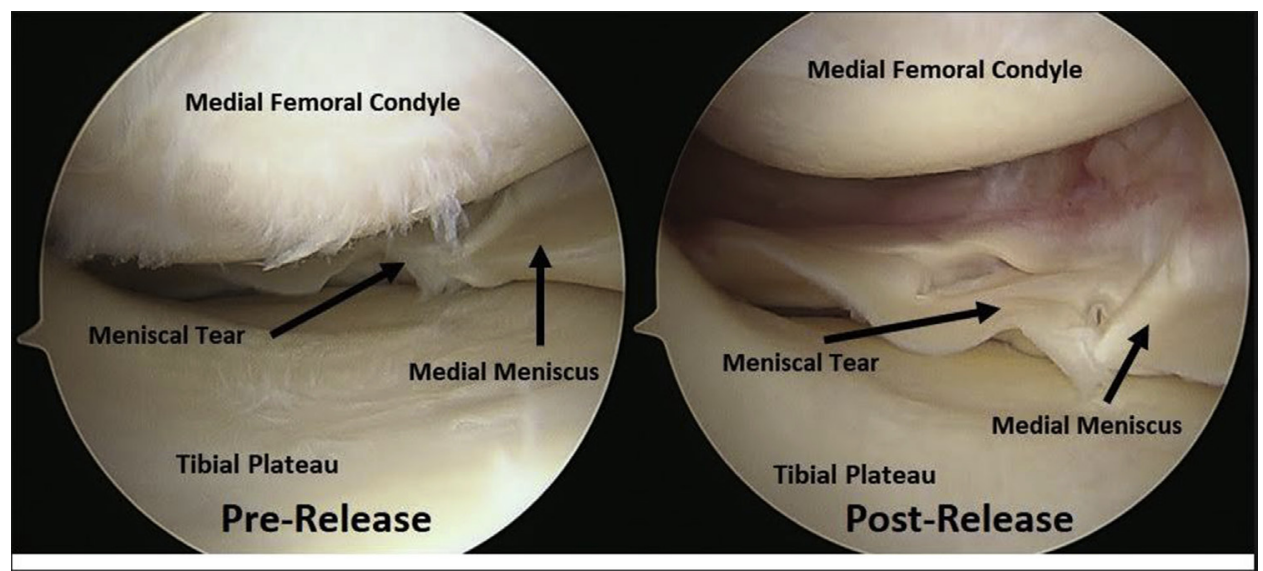




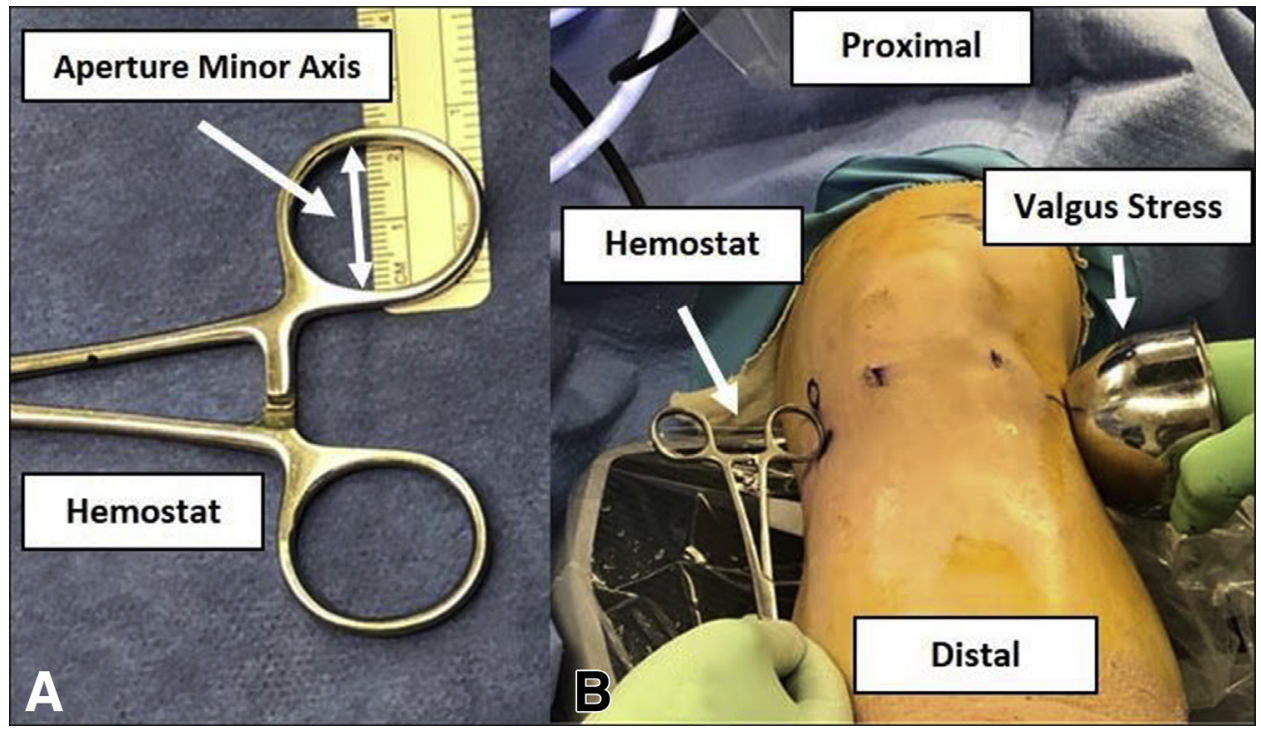

Fig 4. (A) A hemostat handle with a known aperture minor axis of $20 \mathrm{~mm}$ was used to ensure consistent measurements of the medial tibiofemoral joint space regardless of the variation in fluoroscopic magnification. (B) Valgus stress is applied to the knee with the hemostat aperture held at the planned level of the release adjacent to the medial compartment. saphenous vein and saphenous nerve injury through physical exam and patient-reported symptoms. At the 6-week follow-up appointment, valgus stress radiographs were acquired using a Telos Stress Device (Telos Arzt-und Krankenhausbedarf, Hungen, Germany) set to a valgus force of 15 decanewtons $(\mathrm{daN})$ as recommended by the system's user manual (Fig 6). ${ }^{15}$ A radiographic $10-\mathrm{cm}$ measurement calibration marker was used as a reference tool to ensure consistent measurement of the medial joint space in these postoperative, valgus-stress radiographs at follow-up (Fig 7).

\section{Statistical Evaluation}

All data from patient questionnaires and surgical reports were recorded in a secure Microsoft Excel spreadsheet (Microsoft Corp., Redmond, WA), and all statistical analyses were performed in SPSS Statistics 26 (IBM, Armonk, NY). Descriptive statistics including mean, standard deviation (SD), median, minimum, and maximum values were calculated for all quantitative variables. Before data collection, a power analysis was performed, showing that 10 patients would be needed to detect a clinically relevant difference of $2 \mathrm{~mm}$ in medial compartmental joint space widening using a paired $t$ test $(\alpha=0.05$, power $=80 \%$ ). This clinically relevant difference was determined from the previous systematic review findings outlined by Moran et. al. ${ }^{12}$ In addition to the comparison of medial joint space width, paired $t$ tests were used to determine differences in IKDC scores, PROMIS scores, flexion, and extension before and after the MCL release. Intention-to-treat analysis was incorporated in the assessment of intraoperative widening afforded by the MCL release to maximize sample size while accounting for patients who were lost to follow-up. A $P$ value $<.05$ was considered statistically significant.

Fig 5. Intra-operative fluoroscopic imaging before (A) and after (B) percutaneous release of the medial collateral ligament (MCL) demonstrates the improvement in visualization afforded by this procedure.

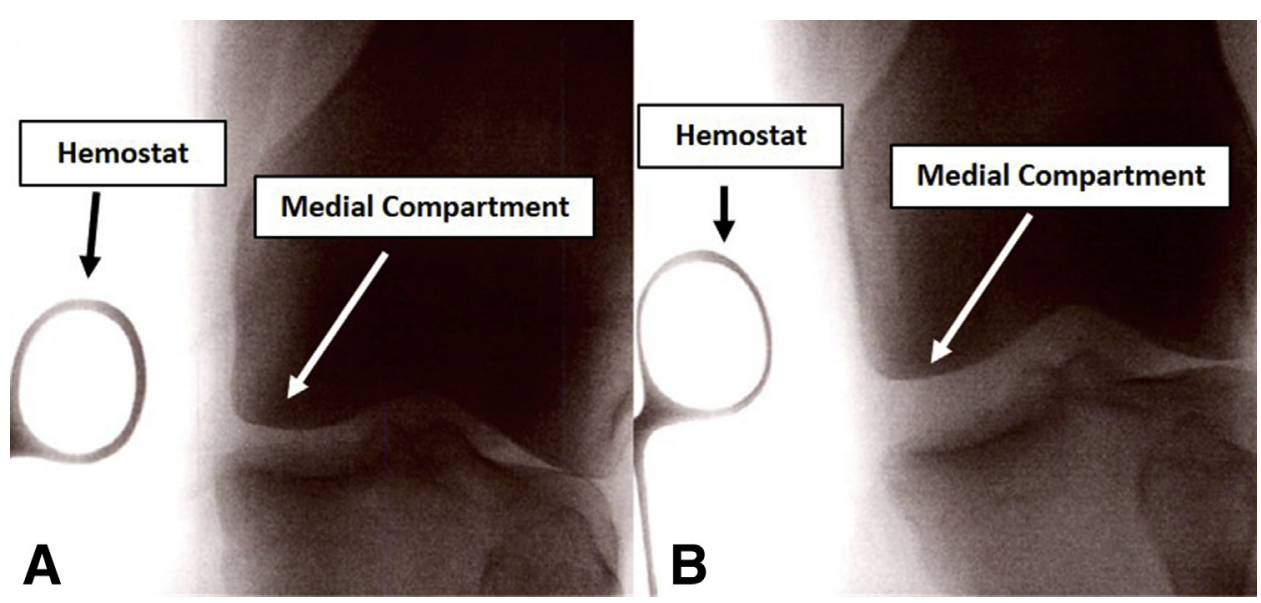


Fig 6. At the 6-week follow-up appointment, a Telos machine is used to provide a consistent valgus force of $15 \mathrm{daN}$ while acquiring stress radiographs of the operative knee to quantify residual medial compartment joint space opening.

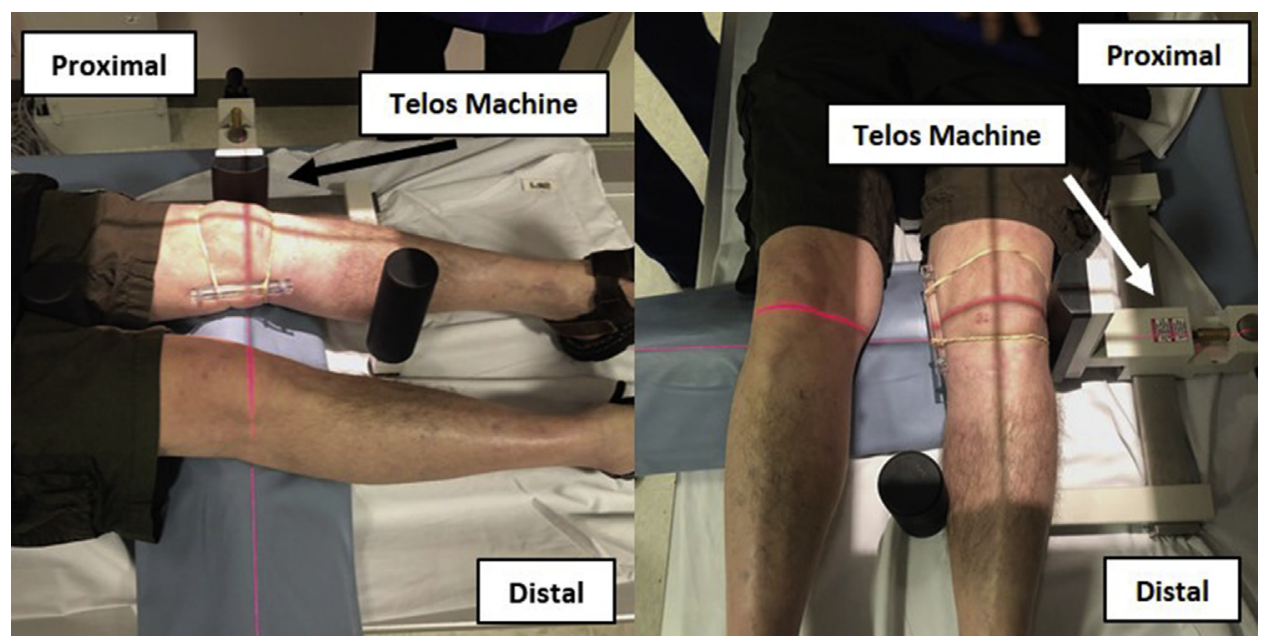

\section{Results}

A total of 59 patients meeting all inclusion criteria provided consent and were enrolled in the prospective study. Seventeen patients were excluded from the final analysis because the MCL release did not need to be performed (adequate space of the medial compartment for visualization and maneuvering of instrumentation) $(n=6)$, surgery cancellations $(n=7)$, and scheduled surgery beyond the end date of the prospective study $(\mathrm{n}=4)$. Through intention-to-treat analysis, 15 patients with either incomplete preoperative or postoperative questionnaires were also included within the analysis of intraoperative measurements. Of patients with recorded intraoperative measurements, 22 were male and 20 were female, with a mean age of $55.3 \pm 10.7$ years (Table 1). Twenty-four of the meniscectomy procedures were performed on the left leg, and 18 were performed on the right (Table 1).
In the evaluation of preoperative range of motion about the knee $(\mathrm{n}=27)$, the mean flexion and extension for participants was quantified as $124.7^{\circ} \pm 8.4^{\circ}$ and $0.6^{\circ} \pm 1.9^{\circ}$, respectively (Table 2). Subjective assessment of each patient's knee functionality produced a mean baseline PROMIS score of $73.9 \pm 12.5$ and a baseline IKDC score of $39.1 \pm 12.7$ (Table 2). No patient enrolled in the study was excluded on the basis of knee laxity after the preoperative valgus stress tests.

Intraoperatively, fluoroscopic imaging of the medial compartment before percutaneous release of the MCL showed a mean width of $5.95 \pm 1.32 \mathrm{~mm}$ (Table 3). After percutaneous release, the average width of the medial compartment was $11.09 \pm 1.74 \mathrm{~mm}$, corresponding to an increase of $5.15 \pm 1.13 \mathrm{~mm}$ (Table 3). This widening of the medial compartment was found to be statistically significant (95\% confidence interval [CI] 4.79 to $5.50, P=.000$ ) (Table 3 ).

\section{Radiographic $10 \mathrm{~cm}$ measurement calibration marker}

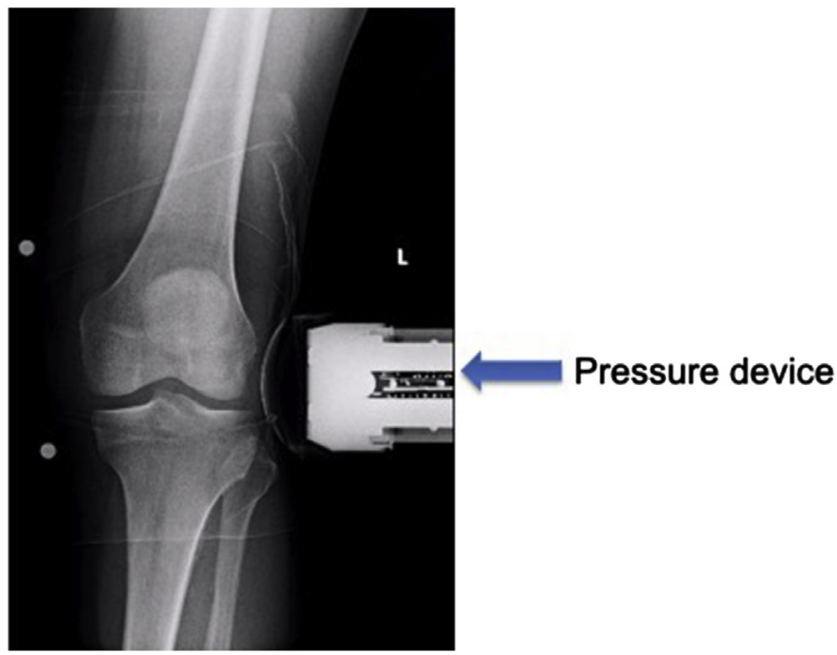

Fig 7. A radiographic 10-cm measurement calibration marker was used as a reference tool to ensure consistent measurement of the medial joint space in postoperative, valgus-stress radiographs at follow-up. 
Table 1. Descriptive statistics of demographics and knee outcomes for patients who underwent medial collateral ligament (MCL) release $(\mathrm{n}=42)$

\begin{tabular}{lc}
\hline \multicolumn{1}{c}{ Characteristic } & Value \\
\hline Age (y) & \\
Mean \pm standard deviation & $55.3 \pm 10.7$ \\
Median (range) & $54.5(31$ to 77$)$ \\
Sex & $20(48)$ \\
Female & $22(52)$ \\
Male & $18(43)$ \\
Laterality & $24(57)$ \\
Right & \\
Left & $42(100)$ \\
Adverse Event & $0(0)$ \\
No & \\
Yes & $7(26)$ \\
Postoperative medial-sided knee pain & $20(74)$ \\
Yes & \\
No &
\end{tabular}

Data are $\mathrm{n}(\%)$ unless noted otherwise.

At the 6-week follow-up appointment $(\mathrm{n}=27)$, reassessment of knee range of motion found the average flexion and extension to be $123.4^{\circ} \pm 10.4^{\circ}$ and $0.4^{\circ} \pm 2.0^{\circ}$, respectively (Table 2 ). This corresponded to a statistically insignificant decrease in flexion of $1.3^{\circ} \pm 10.9^{\circ}(\mathrm{CI}-5.6$ to $3.0, P=.541)$ and a statistically insignificant increase in extension of $0.2^{\circ} \pm 2.7^{\circ}$ (CI -1.3 to $0.9, P=.725$ ) (Table 2 ). Six-week postoperative PROMIS and IKDC scores reported a mean of $80.8 \pm 14.3$ and $50.9 \pm 16.3$, respectively (Table 2 ). Thus, the PROMIS score was found to have a statistically significant increase of $6.9 \pm 12.4$ (CI 2.0 to 11.8 , $P=.008$ ) and the IKDC score, a significant increase of $11.7 \pm 17.8$ (CI 4.7 to $18.8, P=.002$ ) (Table 2). Radiographic evaluation of the medial compartment with valgus stress for the 33 patients in whom followup imaging was available illustrated a mean width of $5.85 \pm 0.99 \mathrm{~mm}$. The difference was not statistically significant compared with before MCL release and represented a mean decrease of $0.18 \pm 1.42 \mathrm{~mm}$ (CI -0.68 to $0.33, P=.474$ ) (Table 3 ). Seven of the 27 patients who completed the postoperative questionnaire reported medial-sided knee pain (Table 1).

\section{Discussion}

In this prospective study, the use of intraoperative, fluoroscopic, valgus-stress radiographs demonstrated that the outside-in, percutaneous release of the superficial MCL intraoperatively on average doubled the medial tibiofemoral joint width, which allowed for improved visualization and facilitated instrumentation in knees with a tight medial tibiofemoral compartment. Importantly, this study also demonstrated radiographic and clinical resolution of any iatrogenic laxity by 6 weeks postoperatively, without the need for postoperative bracing after the release performed during arthroscopic partial medial meniscectomy.

Perhaps the most important finding from this study is the quantification of the increase in medial tibiofemoral joint space intraoperatively, further adding to previous literature attempting to quantify compartment widening. In a study of 10 cadaver knees, Roussignol et al. ${ }^{16}$ reported a mean $3.9-\mathrm{mm}$ increase in the medial tibiofemoral joint space immediately after percutaneous MCL release. Another study by Fakioglu et al. ${ }^{6}$ reported a median $2.0-\mathrm{mm}$ increase in the medial tibiofemoral joint space at 1 week postoperatively after percutaneous release of the MCL during knee arthroscopy in 18 patients. Aside from the current study, intraoperative imaging used to quantify medial compartment widening provided by release of the MCL has been reported only in a study by Polat et al. ${ }^{13}$ In a prospective study of 18 patients, they described a mean medial joint space of $5.1 \mathrm{~mm}$ before application of valgus stress, $7.8 \mathrm{~mm}$ with the application of valgus

Table 2. Preoperative and postoperative knee functionality scores and range of motion for patients who underwent medial collateral ligament $(\mathrm{MCL})$ release $(\mathrm{n}=27)$

\begin{tabular}{|c|c|c|c|c|c|}
\hline Measure & Minimum & Maximum & Median & Mean \pm SD & $P$ Value \\
\hline \multicolumn{6}{|l|}{ IKDC } \\
\hline Preoperative & 11 & 65 & 40 & $39.1 \pm 12.7$ & \\
\hline Postoperative & 16 & 81 & 51 & $50.9 \pm 16.3$ & $.002^{*}$ \\
\hline Preoperative & 46 & 93 & 74 & $73.9 \pm 12.5$ & \\
\hline Postoperative & 37 & 99 & 83 & $80.8 \pm 14.3$ & $.008^{*}$ \\
\hline \multicolumn{6}{|l|}{ Flexion $\left({ }^{\circ}\right)$} \\
\hline \multicolumn{6}{|l|}{ Extension $\left({ }^{\circ}\right)$} \\
\hline Preoperative & -3 & 5 & 0 & $0.6 \pm 1.9$ & \\
\hline Postoperative & -1 & 10 & 0 & $0.4 \pm 2.0$ & .725 \\
\hline
\end{tabular}

IKDC, International Knee Documentation Committee; PROMIS, Patient-Reported Outcomes Measurement Information System; SD, standard deviation.

*Statistically significant $(\alpha=0.05)$. 
Table 3. Fluoroscopic intraoperative measurements of medial compartment width before and after medial collateral ligament $(\mathrm{MCL})$ release $(\mathrm{n}=42)$ and radiographic compartment width at 6-week follow-up $(\mathrm{n}=33)$

\begin{tabular}{lcrrr}
\hline \multicolumn{1}{c}{ Measure } & Minimum & Maximum & Median & Mean \pm SD \\
\hline Medial compartment joint space width (mm) & & & & \\
Before release & 3.10 & 9.44 & 5.93 & $5.95 \pm 1.32$ \\
After release & 8.00 & 14.40 & 11.01 & $11.09 \pm 1.74$ \\
Extent of widening & 3.08 & 8.60 & 5.17 & $5.15 \pm 1.13$ \\
6-wk follow-up & 4.10 & 8.19 & 5.70 & $5.85 \pm .99$ \\
\hline
\end{tabular}

SD, standard deviation.

*Statistically significant $(\alpha=0.05)$

stress, and $12.5 \mathrm{~mm}$ immediately after percutaneous release of the MCL. The authors concluded that release provided $\sim 2.45 \times$ wider visualization of the medial joint space (or $\sim 1.6 \times$ wider visualization using the baseline valgus force measurement). ${ }^{13}$

This study has several key differences from previous studies, however. With 42 patients available for analysis, the present study is better equipped with a larger sample size to draw conclusions regarding quantification of the increase in joint space. The finding that this release provided a mean $1.91 \times$ wider working space within the medial tibiofemoral joint, however, is relatively consistent with the study by Polat et al. ${ }^{13}$ Additionally, this study is the only one to drain fluid from the knee before acquiring intraoperative radiographs and measurements, potentially leading to more accurate and representative measurements. The described release also took place just proximal to the joint line, in comparison to below the joint line in the study by Polat et al. ${ }^{13}$ Performance of the release at the proximal superficial MCL permits transillumination of the joint line to ensure avoidance of the greater saphenous vein and saphenous nerve, while also allowing for direct intra-articular visualization of the release site to confirm adequacy of the release and monitor the act of fiber fenestration. Other major differences in comparison to the study by Polat et al. ${ }^{13}$ are that the current study did not use postoperative bracing and did perform follow-up, valgus-stress radiographs to demonstrate resolution of any iatrogenic laxity.

By providing adequate radiographic follow-up, this study successfully demonstrated both the effectiveness of the percutaneous MCL release by quantifying the additional joint space provided by the release and the subsequent resolution of clinical and radiographic iatrogenic laxity at 6 weeks postoperatively. Zhu et al. ${ }^{11}$ reported a $1.2-\mathrm{mm}$ greater medial joint space compared with baseline at 4 weeks postoperatively. Lons et al. ${ }^{10}$ reported a $1.1-\mathrm{mm}$ greater medial joint space at 6 weeks postoperatively. Neither study quantified the immediate release, however, which makes the temporality and full magnitude of resolution of iatrogenic laxity unclear.

Additionally, the present study found a statistically nonsignificant decrease in the joint space compared with baseline, which contradicts the expected return or slight increase of the medial compartment width with respect to baseline. This finding could be a result of measurement-associated error; however, Jeon et al. ${ }^{7}$ reported a similar phenomenon, with a mean decrease of $0.3 \mathrm{~mm}$ in joint space at 24 -month followup. Future studies will need to address whether this is a function of the healing process after MCL release or errors associated with measurement methodology.

Because the variable impact of different magnitudes of applied valgus stress on the native width of the medial compartment, a consistent value needed be selected to prevent confounding of the quantified widening provided by the MCL release. Fakioglu et al. ${ }^{6}$ attempted to address this potential variable by applying a constant valgus load of $11 \mathrm{~kg}$-force at postoperative radiographic assessment to account for the loads applied by surgeons intraoperatively. ${ }^{6}$ In their approximation of intraoperative loads, 2 surgeons determined that $11 \mathrm{~kg}$-force of valgus stress was the subjective limit at which the medial compartment could be best visualized without causing iatrogenic injury to the MCL, and thus the approximate load conferred to the joint intraoperatively. ${ }^{6}$ In the present study, objective evaluation of the medial compartment widening achieved by the 15-daN load recommended by the Telos user manual produced similar results in comparison to manual valgus loads placed on the knee by the senior author (M.D.M.) during the development of the followup radiograph protocol. ${ }^{15}$ Furthermore, there was no objective difference in the medial compartment widening achieved between 15 and 20 daN. The authors of the present study combined these observations and literature findings to select 15 daN $(\sim 15.3 \mathrm{~kg}$ force) as the load applied to the knee in postoperative radiographs to account for the approximate stresses placed on the joint during intraoperative performance of the release and acquisition of fluoroscopic images.

Although the power of PROMIS and IKDC scores was not assessed, the comparison of IKDC and PROMIS scores in this study is still able to provide value in demonstrating positive trends in functional outcome scores after percutaneous MCL release. In the context of minimal clinically important differences (MCID), the increases in both IKDC and PROMIS scores suggest that 
the percutaneous MCL release does not have a negative impact on patient perception of knee functionality. Despite the absence of specific MCID values secondary to the confounding effects of a concomitant meniscectomy procedure, the described release of the MCL can be seen as a worthwhile intervention for decreasing the risk of iatrogenic damage to articular cartilage through increasing the width of the medial compartment, while not having a negative effect on patient perceptions of knee functionality postoperatively.

There currently exists a lack of consensus in the literature regarding the decision to brace knees after percutaneous MCL release, and the decision to do so has been primarily dependent on surgeon preference. In studies that opted to perform postoperative bracing, the authors largely acknowledged their decision to be precautionary, as no research clearly demonstrates bracing to be necessary. $6,7,13,17$ One study by Claret et al. $^{8}$ reported no use of postoperative bracing following percutaneous MCL release and found no resultant adverse effects. Additionally, Lyu ${ }^{18}$ performed a larger decompressive medial or posteromedial release in the treatment of medial compartment osteoarthritis and did not perform postoperative bracing, with satisfactory outcome. In all, it appears that although it is reasonable to perform postoperative bracing, it is not necessary to achieve good functional outcome after surgery. In a systematic review by Moran et al., ${ }^{12}$ it was suggested that bracing was not necessary to obtain positive outcomes after MCL release and that a need exists for further examination of the functional outcomes in patients following the use of this technique in the absence of bracing. This study is well-suited for adding to this discussion, as the absence of bracing is the standard of care at the senior author's institution. At 6 months postoperatively, Claret et al. ${ }^{8}$ reported no significant difference in Lysholm and Tegner scores between a cohort undergoing percutaneous MCL release during knee arthroscopy and a group undergoing the same procedure without release. Neither group received postoperative bracing. ${ }^{8}$ By comparison, this study reports improved patient-reported functional outcome measures compared with baseline and resolution of laxity at 6 weeks postoperatively after percutaneous release in the absence of postoperative bracing.

Finally, in addition to demonstrating the resolution of residual valgus laxity and improvement in patientperceived functional outcomes at 6 weeks after percutaneous release of the MCL, this study did not detect any injury to the saphenous nerve or greater saphenous vein. Although this is a theoretical risk given the location of the release, a systematic review by Moran et al. $^{12}$ reported no occurrences of such an injury occurring in 202 included patients. Cumulatively, this appears to be a consideration when performing the release, but represents little actual risk. However, despite an absence of evidence for iatrogenic injury, 7 patients reported medial-sided knee pain after release, at 6-week follow-up. Of these patients, 6 reported medial-sided knee pain preoperatively, therefore making it difficult to attribute postoperative pain to the performance of the MCL release.

Future areas of study on this topic could include a similar study that also compares functional outcome scores in patients undergoing this percutaneous release with that of a control group, or examining resolution of iatrogenic laxity after percutaneous release during meniscal repair. It is possible that a larger opening of the medial joint space is required to facilitate suture passage than is needed for meniscectomy alone, and that this would require a greater length of time to reliably return to baseline.

\section{Limitations}

The major limitation of this study was the absence of a validated measurement protocol for assessing width of the medial compartment joint space intraoperatively. Although the use of the hemostat aperture minor axis as a reference and the manual measurement of intraoperative fluoroscopic images before and after the release provided a convenient and resource-limited method of assessing joint widening, there was the potential for error to be introduced at several points. Namely, the use of the hemostat is a nonvalidated reference technique and introduces the risk of slight rotation during the acquisition of valgus stress fluoroscopic images, thus altering the perceived minor axis length during measurement. Furthermore, the use of manual measurements using the printed fluoroscopic images presents the risk for error due the lack of demonstrated intra- and interobserver reliability, as only the senior author (M.D.M.) performed the measurements. This could be seen to introduce bias into the measurements. Additionally, the intraoperative valgus stress views present the potential for error in quantifying intraoperative joint space widening because of the absence of studies validating this method of applying valgus stress and the inability to ensure a consistent valgus force was being applied for every image. The inability to ensure perfect alignment of the medial compartment during intraoperative fluoroscopy could also affect the measurement points for the printed fluoroscopic images. Collectively, these factors could impact the precision and margin for error upon calculation of the medial tibiofemoral joint space; however, using similar methodology for measurement, and having a greater sample size in comparison to other studies examining this outcome in the literature, reduces the chance for the introduction of error. Another limitation of this study was the selection of 15 daN as the valgus load applied to acquire stress 
radiographs postoperatively. This force is approximately $15.3 \mathrm{~kg}$-force and is therefore higher than previous estimates of valgus force placed on the knee during the acquisition of valgus stress images. ${ }^{6}$ With a higher force being applied to the knee in postoperative imaging, it can be reasoned that a higher magnitude of craniocaudal strain is being placed on the MCL, thus contributing to an underestimation of the degree of resolved laxity in this study due to the artificially increased width of the medial compartment.

The method by which postoperative images were obtained differs from the method by which intraoperative images were acquired, which could further complicate comparisons between intraoperative joint space widening and resolution of laxity at 6-week follow-up. This case series was unable to compare preoperative and postoperative IKDC and PROMIS scores for 15 patients as a result of incomplete patient questionnaires. Also, 9 patients did not receive followup stress radiographs secondary to 6 patients being lost to follow-up and 3 patients being unable to schedule 6-week follow-up appointments owing to clinical disruptions caused by COVID-19. As such, functional outcome scores and valgus stress radiographs at 6 weeks postoperatively were unable to be acquired. It cannot be ruled out that there was a systematic reason for loss of follow-up that could have impacted the conclusions drawn by this study.

In comparison to previous research, this study could be limited by a short length of follow-up of 6 weeks as opposed to 24 months, as previously noted. Further studies are needed to adequately assess if this is an inherent limitation of the study or a follow-up consistent with confirming resolution of laxity after MCL release. Finally, because of the lack of normative data for PROMIS and IKDC, a power analysis was unable to be accurately performed for these secondary outcomes before this study, thus limiting assessment of the MCL release's impact on patient perceived knee functionality postoperatively.

Despite these limitations, this study remains a valuable addition to the literature, as it both demonstrates this technique to reliably afford surgeons $\sim 2 \times$ wider working space within the medial tibiofemoral joint, and provides consistent functional outcomes and resolution of any iatrogenic laxity by 6 weeks postoperatively without need for postoperative bracing.

\section{Conclusions}

Percutaneous MCL release during knee arthroscopy effectively improves visualization and facilitates instrumentation by providing an almost $2 \times$ wider working space within the working space of the medial tibiofemoral joint. In this study, the performance of percutaneous MCL release did not result in any complications. Radiographic and clinical resolution of iatrogenic laxity is demonstrated by 6 weeks postoperatively, without the use of postoperative bracing.

\section{Acknowledgments}

Wendy Novicoff, PhD, provided assistance in performance of statistical analysis.

\section{References}

1. Mordecai SC, Al-Hadithy N, Ware HE, Gupte CM. Treatment of meniscal tears: An evidence based approach. World J Orthop 2014;5:233-241.

2. Ward BD, Lubowitz JH. Basic knee arthroscopy Part 3: Diagnostic arthroscopy. Arthrosc Tech 2013;2:e503-e505.

3. Dick W, Glinz W, Henche HR, Ruckstuhl J, Wruhs O, Zollinger $\mathrm{H}$. Complications of arthroscopy. A review of 3714 cases. Arch Orthop Trauma Surg 1978;92:69-73 [in German].

4. Sonnery-Cottet B, Conteduca J, Thaunat M, Gunepin FX, Seil R. Hidden lesions of the posterior horn of the medial meniscus: A systematic arthroscopic exploration of the concealed portion of the knee. Am J Sports Med 2014;42: 921-926.

5. Nielsen DM, Twyman R. Arthroscopic visualization of the posterior horn of the medial meniscus. Arthroscopy 2005;21:1272.el-1272.e2.

6. Fakioglu O, Ozsoy MH, Ozdemir HM, Yigit $\mathrm{H}$, Cavusoglu AT, Lobenhoffer P. Percutaneous medial collateral ligament release in arthroscopic medial meniscectomy in tight knees. Knee Surg Sports Traumatol Arthrosc 2013;21:1540-1545.

7. Jeon SW, Jung M, Chun YM, et al. The percutaneous piecrusting medial release during arthroscopic procedures of the medial meniscus does neither affect valgus laxity nor clinical outcome. Knee Surg Sports Traumatol Arthrosc 2018;26:2912-2919.

8. Claret G, Montañana J, Rios J, et al. The effect of percutaneous release of the medial collateral ligament in arthroscopic medial meniscectomy on functional outcome. Knee 2016;23:251-255.

9. Javidan P, Ahmed M, Kaar SG. Arthroscopic release of the deep medial collateral ligament to assist in exposure of the medial tibiofemoral compartment. Arthrosc Tech 2014;3: e699-e701.

10. Lons A, Boureau F, Drumez E, Pasquier G, Putman S. Does medial collateral ligament pie-crusting induce residual laxity in arthroscopic management of medial meniscus tears? A prospective study of 40 cases. Orthop Traumatol Surg Res 2018;104:707-711.

11. Zhu W, Tang Q, Liao L, Li D, Yang Y, Chen Y. Application of pie-crusting the medial collateral ligament release in arthroscopic surgery for posterior horn of medial meniscus in knee joint. Zhong Nan Da Xue Xue Bao Yi Xue Ban 2017;42:1053-1057 [in Chinese].

12. Moran TE, Awowale JT, Werner BC, Fox MA, Miller MD. Associated morbidity after the percutaneous release of the medial collateral ligament for knee arthroscopy. J Arthrosc 2020;36:891-900.

13. Polat B, Deniz A, Polat AE, et al. Objective measurement of medial joint space widening with percutaneous 'pie 


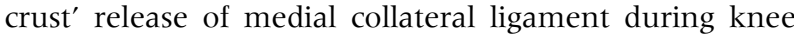
arthroscopy. J Knee Surg 20120;33:94-98.

14. Collins NJ, Misra D. Measures of knee function. Arthritis Care Res (Hoboken) 2011;63:S208-S228 (suppl 11).

15. Scheuba G. Operating instructions Telos-Stress-Device GA-III/E. Available at: http://www.telosmedical.com/ library/public/documents/PDFs/thelos-device.pdf 2009. Hungen, Germany: Telos Arzt-und Krankenhausbedarf.

16. Roussignol X, Gauthe R, Rahali S, Mandereau C, Courage O, Duparc F. Opening the medial tibiofemoral compartment by pie-crusting the superficial medial collateral ligament at its tibial insertion: A cadaver study. Orthop Traumatol Surg Res 2015;101:529-533.

17. Chung KS, Ha JK, Ra HJ, Kim JG. Does release of the superficial medial collateral ligament result in clinically harmful effects after the fixation of medial meniscus posterior root tears? Arthroscopy 2017;33: 199-208.

18. Lyu SR. Arthroscopic medial release for medial compartment osteoarthritis of the knee: The result of a single surgeon series with a minimum follow-up of four years. J Bone Joint Surg Br 2008;90:1186-1192. 